\title{
Molecular diagnosis of Ewing sarcoma/ primitive neuroectodermal tumor in routinely processed tissue: a comparison of two FISH strategies and RT-PCR in malignant round cell tumors
}

\author{
Robert S Bridge, Veena Rajaram, Louis P Dehner, John D Pfeifer and Arie Perry \\ Department of Pathology and Immunology, Lauren V Ackerman Laboratory of Surgical Pathology, \\ Barnes-Jewish and St Louis Children's Hospitals, Washington University Medical Center, St Louis, \\ $M O, U S A$
}

\begin{abstract}
Ewing sarcoma/primitive neuroectodermal tumor (EWS/PNET) is a diagnostically challenging malignant round cell tumor with signature translocations involving the EWS gene. These translocations are detectable with both reverse transcriptase-polymerase chain reaction (RT-PCR) and fluorescence in situ hybridization (FISH) in formalin-fixed paraffin-embedded tissue. However, RT-PCR is less sensitive in formalin-fixed paraffinembedded than frozen tissue. Similarly, commercial FISH probes have recently become available, but have yet to be rigorously tested in the clinical setting. Therefore, we have compared RT-PCR with FISH using 'home brew' fusion probes for Ewing sarcoma (EWS)-FLI1 and a commercial EWS break apart probe set in 67 archival round cell tumors, including 27 EWS/PNETs. Sensitivities and specificities for both FISH assays were 91 and $100 \%$, respectively, whereas RT-PCR had a sensitivity of $54 \%$ and a specificity of $85 \%$. The break apart strategy was easier to interpret than probe fusion approach. We conclude that FISH is a more sensitive and reliable ancillary technique than RT-PCR for the diagnosis of EWS/PNET in formalin-fixed paraffin-embedded tissue, although the latter provides additional information regarding fusion transcript subtype and prognosis. The commercial break apart probe set is both readily available and easy to interpret, making it particularly attractive. Nonetheless, complex round cell tumors often benefit from molecular testing with multiple methods.
\end{abstract}

Modern Pathology (2006) 19, 1-8. doi:10.1038/modpathol.3800486; publilshed online 2 September 2005

Keywords: Ewing sarcoma; FISH; fusion transcript; molecular diagnostics; primitive neuroectodermal tumor; RT-PCR; translocation

Ewing sarcoma/peripheral primitive neuroectodermal tumor (EWS/PNET) is the prototype within the category of EWS family tumors. ${ }^{1}$ The relationship between these tumor types has been studied extensively using immunohistochemical, genetic, and ultrastructural techniques leading to our current understanding of EWS/PNET as a highly cellular and monotonous primitive appearing neoplasm with a limited potential for neuronal differentiation, typically arising in the bone or soft tissue of individuals in the first few decades of life. ${ }^{2}$ The

Correspondence: Dr A Perry, MD, Department of Pathology and Immunology, Box 8118, Washington University School of Medicine, 660 S. Euclid Ave., St Louis, MO 63110-1093, USA. E-mail: aperry@wustl.edu

Received 6 July 2005; revised 28 July 2005; accepted 1 August 2005; published online 2 September 2005
EWS/PNET has overlapping morphologic features with other round cell tumors of childhood and adolescence. For prognosis and appropriate management, it is important to differentiate EWS/PNET from classic neuroblastoma, Wilms tumor, and rhabdomyosarcoma in particular. We have also come to recognize the fact that there exists a category of undifferentiated malignant round cell tumor, often designated as 'undifferentiated round cell sarcoma', which fails current diagnostic strategies for a more definitive classification.

Recent developments in molecular pathology have greatly enhanced our ability to classify round cell tumors and in the case of EWS/PNET, most tumors have a balanced translocation involving chromosomes 11 and 22, which fuse portions of the EWS gene on $22 \mathrm{q} 12$ with the FLI1 gene on 11q24, thus creating a novel fusion gene with oncogenic 
properties. $^{3-8}$ A number of variant translocations have been described as well, most of which result in fusions between EWS and other members of the ETS family of transcriptional regulators, such as $E R G$ on 21q22, ETV1 on 7p22, E1AF on 17q12, and FEV on 2q33. Rare cases harboring FUS-ERG fusion transcripts have also been reported. ${ }^{9}$ These translocations help distinguish EWS/PNET from other round cell tumors and are therefore, diagnostically useful. Reverse transcription polymerase chain reaction (RT-PCR) has been used most commonly to identify the precise fusion transcripts and the specific chromosomal breakpoints involved in the translocation. ${ }^{10-12}$ In the setting of diagnostic surgical pathology, however, particularly with small biopsies and outside consultation cases, fresh frozen tissue is often not available. Whereas both RT-PCR and fluorescence in situ hybridization (FISH) have been successfully applied to routine formalin-fixed paraffin-embedded specimens, the former may have limited utility because of poor ribonucleic acid (RNA) quality, which is associated with diminished sensitivity when compared with frozen tissue. Similarly, both break apart deoxyribonucleic acid (DNA) probes flanking the EWS breakpoint region on 22q12 and EWS-FLI1 fusion probes have been previously utilized for FISH analysis to detect the presence of the translocation, but they have predominantly been applied to fresh frozen or cytologic material. ${ }^{13-18}$ Recently, a FISH break apart probe set has become commercially available, although it has yet to be rigorously tested in formalin-fixed paraffinembedded specimens in the clinical setting. In the current study, we compared the diagnostic utility of FISH fusion strategy with 'home brew' probes, FISH break apart strategy with commercial probes, and RT-PCR on a series of round cell tumors, including EWS/PNET, alveolar rhabdomyosarcoma, neuroblastoma, small cell carcinoma, and undifferentiated round cell sarcoma, not otherwise specified.

\section{Materials and methods}

\section{Tumor Cohort}

With local Institutional Review Board (IRB) approval, archival blocks or unstained sections from consult cases from 67 consecutive nonhematopoietic round cell tumors biopsied or resected from May 1998 to August 2004 were reviewed and retrieved for further study from the surgical files of the Lauren V. Ackerman Laboratory of Surgical Pathology, Barnes-Jewish and St Louis Children's Hospitals, Washington University Medical Center. The study set consisted of 27 EWS/PNETs, 11 undifferentiated round cell sarcomas, 10 small cell carcinomas, eight neuroblastomas, seven alveolar rhabdomyosarcomas, two fibrosarcomas in infants with cellular round cell foci, and one malignant teratoma with a PNET-like component. In most cases, the final diagnosis was established on the basis of clinico- pathologic and immunohistochemical features alone, and for the purposes of this study, the histopathologic diagnosis was utilized as the diagnostic gold standard. Therefore, primitive round cell tumors whose histologic and immunohistochemical features were not diagnostic for any single established entity were assigned to the undifferentiated round cell sarcoma category, so that determinations of testing sensitivities and specificities would not be skewed by these more ambiguous or indeterminate cases. In other words, tumors with specific diagnoses were histopathologically classic examples, whereas nonclassic cases were assigned to the undifferentiated round cell sarcoma category. In addition to routine histology and immunohistochemistry, 30 tumors (45\%) had been previously studied with ancillary molecular diagnostic techniques, predominantly RT-PCR. This was particularly common for outside consults and the undifferentiated round cell sarcoma category.

\section{Fluorescence In Situ Hybridization}

The FISH assay was performed as previously reported..$^{19}$ Sections from representative blocks were cut onto positively charged slides at $5 \mu \mathrm{m}$ thickness. Deparaffinization of the sections was carried out with two 10-min immersions in Citrisolv, followed by three 3 -min immersions in isopropanol. The slides were next rinsed in running water for $5 \mathrm{~min}$, followed by distilled water for $3 \mathrm{~min}$. Antigen retrieval was achieved by immersing the slides in citrate buffer ( $\mathrm{pH}$ 6.0) within a plastic Coplin jar, which was steamed a total of $20 \mathrm{~min}$, then slowly cooled to room temperature. The slides were then rinsed in running water for $5 \mathrm{~min}$, followed by distilled water for $3 \mathrm{~min}$. The sections were subjected to $0.4 \%$ pepsin (P-7012, Sigma-Aldrich, St Louis, MO, USA) digestion for $15 \mathrm{~min}$ at $37^{\circ} \mathrm{C}$, and then were placed in $2 \times$ standard saline citrate (SSC) on a rotator for $5 \mathrm{~min}$. Slides were then airdried. For the FISH break apart strategy, the commercial EWSR1 dual color break apart set was utilized (Vysis Inc., Downers Grove, IL, USA). It combines a $500 \mathrm{~kb}$ Spectrum Orange-labeled probe on the centromeric side of the $7 \mathrm{~kb}$ EWSR1 breakpoint region between exons 7 and 10 of the EWS gene with an $1100 \mathrm{~kb}$ Spectrum Green-labeled probe localizing just telomeric to this breakpoint region (http://www.vysis.com). For the FISH fusion approach, a paired set of 'home brew' rhodaminelabeled EWS and fluoroisothiocyanate (FITC)-labeled FLI-1 were utilized (gift from $\mathrm{Dr}$ Paul Meltzer) ${ }^{20}$ Each set of paired probes were diluted from stock with tDenHybTM hybridization buffer (Insitus Biotechnologies, Albuquerque, NM, USA) to a 1:25 working concentration, with $10 \mu \mathrm{l}$ dispensed per slide. Codenaturation was achieved by placing slides upon the metal surface of a light-shielded slide moat (Boekel scientific slide moat, model 240000) preheated to $90^{\circ} \mathrm{C}$ for $13 \mathrm{~min}$. Slides were 
hybridized overnight in a $37^{\circ} \mathrm{C}$ humidified chamber. The next day, slides were washed in $50 \%$ formamide/ $1 \times$ SSC solution, followed by two washes of SSC for $2 \mathrm{~min}$ each. Slides were removed and allowed to air dry. 4',6-Diamidino-2-phenylindole (DAPI) $(10 \mu \mathrm{l})$ in Fluorgard (Insitus) was applied to each of the slides, which were then coverslipped. Green and red fluorescent signals were enumerated in regions of cellular tumor under an Olympus BX60 fluorescence microscope with appropriate filters (Olympus, Melville, NY, USA).

For each hybridization, a minimum of 100 nonoverlapping nuclei were assessed for the presence of fused and/or split green and red signals. In most cases, two or three of the authors (RSB, VR, AP) each enumerated the hybridizations yielding cumulative counts of 200-300 cells. However, the minimum number of 100 cells assessed per case was arbitrarily chosen, since there are currently no universally accepted consensus criteria published. Prior investigators have utilized counts ranging from 20 to 200 cell enumerations. ${ }^{13-18,20}$ For the FISH fusion approach, a positive interpretation was defined as $>30 \%$ nuclei with fused signals (yellow signals or paired red and green signals $<1$ signal diameter from one another). For the FISH break apart approach, $>15 \%$ had to have split signals $(>1$ signal diameter apart) for a positive result. These cutoffs were based on counts in non-neoplastic controls (mean +3 s.d.). Hybridizations where signals were either lacking or too weak to be interpreted were repeated using higher probe concentrations. Those still uninterpretable were considered 'noninformative'. FISH images were captured using a black and white, high-resolution COHU charge coupled device (CCD) camera, $Z$-stack motor and CytoVision ${ }^{\mathrm{TM}}$ basic workstation (Applied Imaging, Santa Clara, CA, USA), with sequential DAPI (one level), FITC (10 levels), and rhodamine (10 levels) filter settings. The resulting images were reconstituted with blue, green, and red pseudocolors using CytoVision ${ }^{\mathrm{TM}}$ software.

\section{Reverse Transcription-Polymerase Chain Reaction}

The RT-PCR assay was performed as previously published. ${ }^{21}$

$R N A$ extraction: Four $10 \mu \mathrm{m}$ sections of tumor grossly dissected from the block based on control $\mathrm{H} \& \mathrm{E}$-stained sections to include mainly areas of viable tumor were deparaffinized using xylene and ethanol washes, and digested in buffer (Tris $\mathrm{pH} 7.5,20 \mathrm{mM}$ ethylenediaminetetraacetic acid (EDTA), 1\% sodium dodecyl sulfate (SDS)) containing $25 \mathrm{mg}$ proteinase $\mathrm{K}$. Total RNA was then extracted from tumor tissue using a modified guanidine isothiocyanate method employing Trizol (Gibco/BRL, Gaithersburg, MD, USA) and treated with DNAse I prior to reverse transcription.

RT-PCR: RNA samples were reverse transcribed using Mulv reverse transcriptase and the Gene Amp
RNA-PCR kit (Perkin-Elmer, Foster City, CA, USA), and nested PCR was performed for EWS-FLI1, EWSERG, EWS-ETV1, EWS-E1AF, EWS-FEV, EWS-ERG, and EWS-WT1 using published primers. ${ }^{21,22}$ The amplified EWS-FLI1 fusion products were visualized by ethidium bromide staining after separation by $2 \%$ agarose gel electrophoresis. In each experiment, RNA from the EWS/PNET cell line SK-ES expressing a type 2 EWS-FLI1 transcript ${ }^{21}$ was assayed in parallel as the positive control for EWSFLI1, -ERG, or -FEV transcripts, and the 3T3/ETV1 cell line expressing a recombinant EWS-ETV1 fusion $^{23}$ was assayed in parallel as the positive control for EWS-E1AF and -ETV1 fusion transcripts. A no input DNA (reagent only) sample and a sham reverse transcriptase-treated (no RT) sample from each case were also assayed in parallel as negative controls. To verify the presence of intact RNA and amplifiable cDNA, each reverse transcriptase reaction product was also assayed with primers for the housekeeping gene $\beta 2$-microglobulin.

DNA sequencing: The PCR products were cloned into vector pCR2.1 using the TA Cloning kit (Invitrogen, Carlsbad, CA, USA), then sequenced using the Taq dideoxy terminator cycle sequencing kit (Applied Biosystems Inc., Foster City, CA, USA) and a fluorescent DNA sequencer (373A, Applied Biosystems Inc.). DNA sequence analysis was facilitated by basic local alignment search tool sequence similarity searches using the National Center for Biotechnology Information database (www.ncbi.nlm.nih.gov/BLAST/).

\section{Determinations of Test Performance}

Sensitivities, specificities, positive predictive values, and negative predictive values were calculated using the final pathologic diagnosis as the gold standard and defined as follows: sensitivity = true positives/(true positives plus false negatives); specificity $=$ true negatives/(true negatives plus false positives); positive predictive value $=$ true positives $/$ (true positives plus false positives); negative predictive value $=$ true negatives $/$ (true negatives plus false negatives). Noninformative, nontested, and undifferentiated round cell sarcoma cases were excluded from these determinations (eg sensitivity of $91 \%$ for 20 of 22 informative EWS/PNET cases with a positive result). Cases of EWS/PNET that were negative for an $E W S$-containing rearrangement by FISH fusion, FISH break apart, or RT-PCR were false negatives, whereas those with a positive result, but a nonEWS/PNET diagnosis were considered false positives. Cases that were positive by one molecular technique and negative by another were considered discordant for the two methods being compared. If both were negative or both were positive, they were considered concordant. Cases with missing data (eg noninformative results) were excluded from determinations of concordance. 


\section{Results}

The sensitivities, specificities, positive predictive values, and negative predictive values for each of the three testing methods (FISH break apart, FISH fusion, and RT-PCR) are summarized in Table 1.

Table 1 Comparison of molecular diagnostic techniques for detection of EWS/PNET

\begin{tabular}{lccc}
\hline & $\begin{array}{c}\text { FISH break } \\
\text { apart (N =67) }\end{array}$ & $\begin{array}{c}\text { FISH fusion } \\
(\mathrm{N}=66)\end{array}$ & $\begin{array}{c}\text { RT-PCR } \\
(\mathrm{N}=43)\end{array}$ \\
\hline Sensitivity & $91 \%$ & $91 \%$ & $54 \%$ \\
Specificity & $100 \%$ & $100 \%$ & $85 \%$ \\
Positive PV & $100 \%$ & $100 \%$ & $78 \%$ \\
Negative PV & $93 \%$ & $93 \%$ & $65 \%$ \\
NI assays & $12 \%$ & $15 \%$ & $16 \%$ \\
URCS & 10 neg., 1 NI & 10 neg., 1 NI & 3 pos., ${ }^{a} 7$ neg. \\
\hline
\end{tabular}

$N=$ total number of cases tested by each of the three techniques; $\mathrm{PV}=$ predictive value; $\mathrm{NI}=$ noninformative; $\mathrm{URCS}=$ undifferentiated round cell sarcoma; neg. = negative; pos. $=$ positive

Concordance between the two FISH techniques $=100 \%$; concordance between FISH method and RT-PCR $=67 \%$.

${ }^{\mathrm{a}}$ EWS-FLI1 fusion in one case, EWS-WT1 fusions in two.
Examples of FISH-positive and -negative results are illustrated in Figure 1. Noninformative cases ranged from 12 to $16 \%$ depending on the method utilized. Eight cases examined by FISH break apart and 10 cases by FISH fusion were considered noninformative due to either weak signals or insufficient tissue. Of the 22 informative EWS/PNETs, 20 cases (91\%) were positive by FISH break apart, FISH fusion, or both. The results were concordant in all 17 cases where both were interpretable (Table 1). Of the two cases of EWS/PNET that were negative by FISH, one was positive by RT-PCR and the other was negative. None of the other tumor types was positive using either set of FISH probes. For cases that were positive using break apart FISH probes, there was on average $79 \%$ cells with split signals (range: 36$96 \%$ ), whereas negative cases averaged $5 \%$ (range: 0-14\%). For the FISH fusion method, fused signals were seen in a mean of $70 \%$ (range: $31-89 \%$ ) in positive and $10 \%$ (range: $1-23 \%$ ) in negative cases. Qualitatively, FISH signals were brighter and larger with the FISH break apart than with the FISH fusion technique.
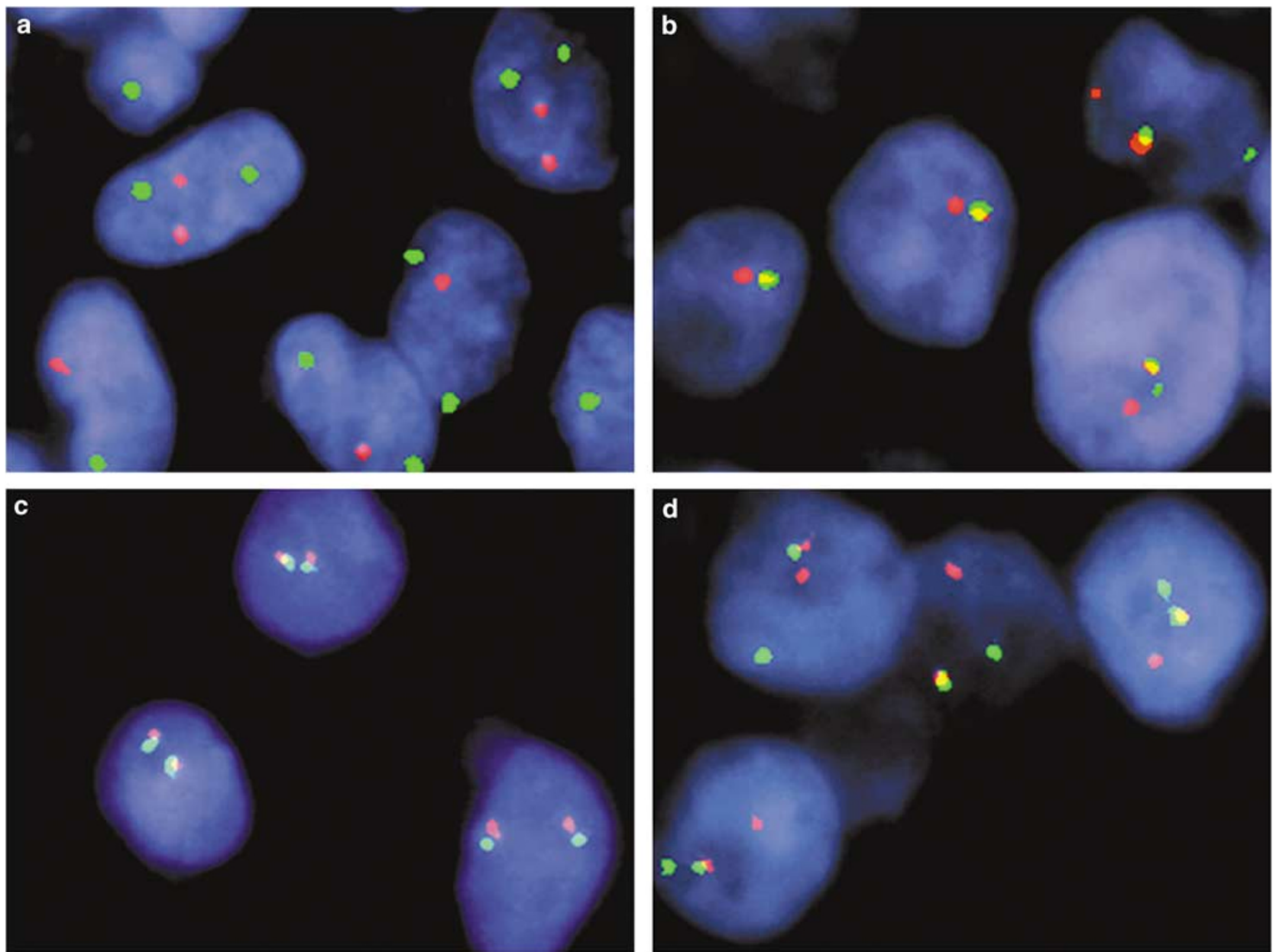

Figure 1 Representative FISH results. (a) FISH-F-negative case of URCS with split EWS (red) and FLI1 (green) signals. (b) FISH-Fpositive EWS/PNET case with fused signals. (c) FISH-BA-negative case of small cell carcinoma with normal fused EWS (centromeric red; telomeric green) signals. (d) FISH-BA-positive EWS/PNET case with split signals in most nuclei. 


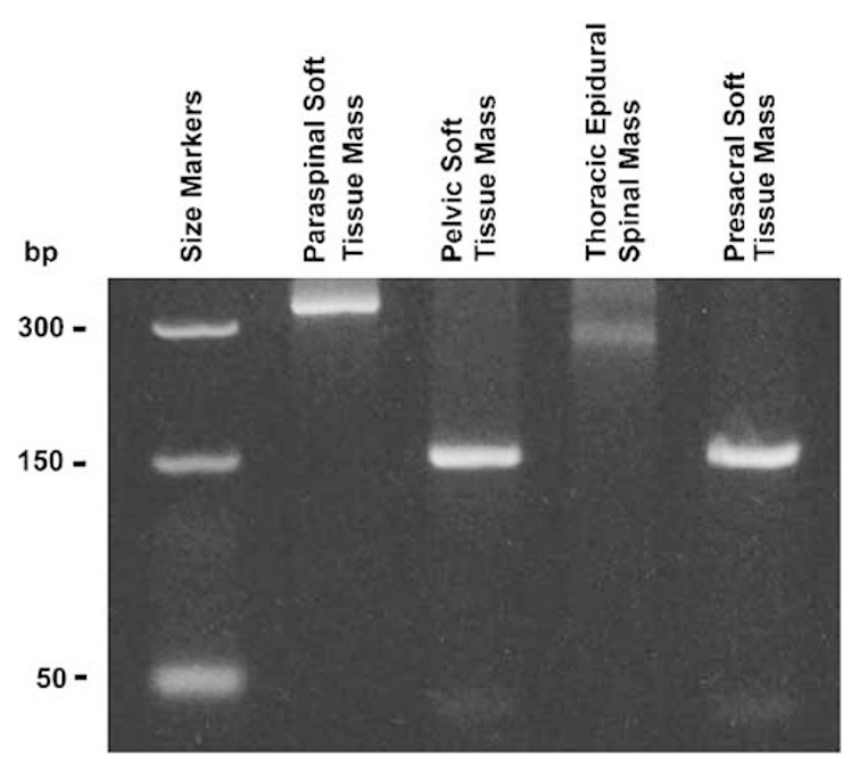

Figure 2 Representative RT-PCR results. RT-PCR analysis of FFPE tissue for EWS-FLI1 fusion transcripts showed a 342 bp band indicative of an EWS exon 7 to FLI1 exon 5 fusion in a paraspinal soft tissue mass from a 17 -year-old girl, a $277 \mathrm{bp}$ band, indicative of an EWS exon 7 to FLI1 exon 6 fusion in a thoracic epidural space mass from a 13-year-old boy, a $150 \mathrm{bp}$ band indicative of an EWS exon 7 to FLI1 exon 8 fusion in the pelvic mass of a 13-yearold boy, and a $150 \mathrm{bp}$ band indicative of an EWS exon 7 to FLI1 exon 8 fusion in the presacral soft tissue mass of a 20-year-old man.

RT-PCR analysis was performed on 43 cases with RNA extracted from formalin-fixed paraffin-embedded blocks (Table 1). Representative results are depicted in Figure 2. Seven cases were noninformative due to a lack of amplifiable RNA. An EWS-FLI1 fusion transcript was identified in nine cases: seven EWS/PNETs, one undifferentiated round cell sarcoma, and one fibrosarcoma with focal round cell elements. An EWS-WT1 fusion was found in two undifferentiated round cell sarcomas. RT-PCR was negative in 12 EWS/PNETs, of which nine were positive by FISH analysis. RT-PCR was concordant with FISH results in $67 \%$ of cases. As previously noted, the most common discordance was due to cases, which were positive by FISH, but negative by RT-PCR. This is consistent with the lower sensitivity of the latter technique in formalin-fixed paraffinembedded tissue (Table 1).

\section{Discussion}

The stratification of malignant round cell tumors into specific subtypes on the basis of histologic and immunohistochemical findings alone is occasionally ambiguous, especially when the experience includes consultation cases. ${ }^{21}$ An expanded panel of immunohistochemical markers is extremely helpful in this diagnostic setting, but nonetheless suffers from variable degrees of insufficient specificities, lack of general availability, and/or technical/interpretive difficulties. ${ }^{24-31}$ Therefore, the development of molecular diagnostic techniques for the detection of relatively unique fusion transcripts present within subtypes of round cell tumors has proven to be a helpful adjunct. However, the reliability of various diagnostic techniques in routine formalin-fixed paraffin-embedded tissue has not been fully explored, especially for those such as the EWS FISH break apart approach, for which DNA probes have only recently become commercially available.

Our data suggest that both the fusion and break apart FISH strategies are highly sensitive and specific techniques for the diagnosis of EWS/PNET. Both successfully detected $90 \%$ of EWS/PNET cases and there were no false positives, correlating well with the $83 \%$ sensitivity recently reported in a recent smaller series. ${ }^{18}$ Interestingly, none of the 11 undifferentiated round cell sarcomas from our files were positive, suggesting that, in addition to EWS/ PNET, they are similarly unlikely to be cases of desmoplastic small round cell tumor (EWS-WT1 fusion transcript), clear cell sarcoma (EWS-ATF1 fusion), or extraskeletal mesenchymal chondrosarcoma (EWS-CHN fusion), since the FISH break apart technique should be positive in the majority of these tumor types as well. ${ }^{32-34}$ Nevertheless, the detection by RT-PCR of EWS-WT1 fusion transcripts in two undifferentiated round cell sarcomas and EWS-FLI1 in another is an intriguing observation. It is unclear whether the discordance in these cases is technical in nature or perhaps represents a minor clone of tumor cells that have developed these translocations as a secondary alteration. Small subsets of such tumor cells could be below the diagnostic threshold of FISH, yet still detectable by RT-PCR. Additional studies are clearly needed to further elucidate the nature of such undifferentiated round cell sarcomas, both with and without detectable fusion transcripts by RT-PCR. Currently, we consider 'undifferentiated round cell sarcoma' to be a nonspecific diagnosis of exclusion, utilized when the tumor does not conform to any of the currently well-characterized categories of round cell sarcoma. Rather than a single entity, it likely represents a heterogeneous collection of primitive tumor types that either have yet to be characterized or are currently recognized forms with atypical clinicopathologic and genetic features.

Of the two FISH probe types tested, the break apart probe set was generally easier to interpret and had the additional advantage of accessibility, given that it is commercial rather than 'homemade', as in the case of the FISH fusion probe set. Additionally, one of the difficulties with the fusion probes is that apposition of signals is normally seen in a subset of nuclei, particularly in densely cellular tumors such as the ones examined in the current study. Therefore, a higher cutoff of nuclei with fused signals $(30 \%)$ was required for the fusion-based FISH strategy than the cutoff for split signals (15\%) used for the break apart technique. Lastly, the commercial probes are particularly large (500 and $1100 \mathrm{~kb})$, 
resulting in enhanced hybridization efficiencies and signal size. In the current study, the FISH break apart yielded interpretable results in $88 \%$ compared with $85 \%$ for the FISH fusion probes. While this difference is negligible, the difference in ease of use and time needed to score was significant. Since all of the noninformative cases in our series were consults, it is also likely that the decreased success rates are in part due to fixation, processing problems, and/or the age of archived slides. For example, our FISH lab has generated interpretable results in roughly $98 \%$ of the last 1400 in-house and consult formalin-fixed paraffin-embedded tumor specimens prospectively analyzed for clinical testing.

Although the two FISH techniques were entirely concordant, a number of discrepancies between FISH and RT-PCR were found, similar to the study by Qian et al. ${ }^{18}$ The overall decreased sensitivity of RT-PCR in formalin-fixed paraffin-embedded tumor specimens has been previously reported, although the $54 \%$ sensitivity of RT-PCR in the current study was considerably lower than the $90 \%$ observed for the two FISH techniques and slightly lower than the $71 \%$ figure previously published from our laboratory for prospectively studied round cell neoplasms. ${ }^{21}$ It is possible that RNA degradation in paraffin blocks and unstained sections archived for several years accounted for this further decline in sensitivity. In this regard, it is also important to note that our control reaction for amplifiable RNA employed $\beta 2$-microglobulin, a transcript that is expressed at significantly higher levels than EWS fusion transcripts. It is therefore possible that we overestimated the number of cases that retained intact amplifiable low-abundance transcripts. However, had we used as a control, a transcript expressed at lower levels, it is likely that more tumors would have been classified as noninformative, artificially increasing the overall sensitivity level of this assay. It is also possible that some of the EWS/PNET cases negative by RT-PCR contained EWS-FLI1 exon combinations that produce very long fusion transcripts not efficiently amplified from formalin-fixed paraffin-embedded tissue, although such fusions appear to be rare based on published series. ${ }^{35}$

Additionally, the RT-PCR data had lower specificity (85 vs 100\%) and lower positive and negative predictive values (78 vs $100 \%$ and 65 vs 93\%, respectively) than FISH. The explanation for the false positives is not readily appreciable in every case, although a number of possibilities exist. As already discussed, RT-PCR is considerably more sensitive than FISH for detecting rare events. Therefore, for the six cases in this study where FISH was negative and RT-PCR was positive, it is possible that there was only a small population of tumor cells containing the fusion transcript. If this is valid, then the interpretation of such a finding is currently unclear. The most common scenario where this was encountered in this series was the undifferentiated round cell sarcoma category, already discussed. Of the remaining three cases, one was in fact diagnosed as EWS/PNET of soft tissue, although the patient's age of 52 years was a little unusual. Nevertheless, the positive EWS-FLI1 RT-PCR result in this case was consistent in all other respects. Another tumor was a malignant teratoma with a PNET-like component in the deep pelvic soft tissues of a 2-year-old boy. The final example was a spindle cell sarcoma with foci of cellular round cell elements in a 3-yearold boy with a positive EWS-FLI1 RT-PCR result. Conventional karyotyping of this same case revealed a 3p26 deletion and a $\mathrm{t}(21 ; 22)(\mathrm{q} 10 ; \mathrm{q} 10)$ translocation, but no evidence of either the classic $t(11 ; 22)(q 24 ; q 12)$ associated with EWS-FLI1 or the $\mathrm{t}(21 ; 22)(\mathrm{q} 22 ; \mathrm{q} 12)$ associated with the EWS-ERG fusions. Unfortunately, none of the other discordant cases had karyotypes performed for comparison with the molecular data. Lastly, technical problems, such as specimen contamination, could also account for false positive results by RT-PCR. Meticulous handling of specimens and appropriate positive and negative controls should minimize this potential pitfall.

While the FISH break apart method provided some clear advantages, its main disadvantage in comparison to FISH fusion and RT-PCR is that the translocation partner is not identified. In this study, there was perfect concordance between the two FISH techniques, consistent with EWS-FLI1 fusions in all of our positive cases. However, for the variant ETS gene family rearrangements that have been previously reported in approximately $10 \%$ of EWS/PNETs (EWS-ERG, EWS-ETV1, EWS-E1AF, and EWS-FEV), the FISH break apart approach would be expected to be positive, whereas the FISH fusion method would not. In contrast to the theoretically higher sensitivity, the potential disadvantage of the FISH break apart approach would be its inability to distinguish EWS/PNET from other tumors that harbor EWS gene rearrangements. With RT-PCR, the decreased sensitivity is off-set by its ability to test for multiple fusion products, thus distinguishing between the various types. Furthermore, there is evidence to suggest that there is additional prognostic information obtained by knowing whether the EWS-FLI1 transcript is type 1 or 2 and this type of information is only available with the RT-PCR method. ${ }^{36,37}$ In the group of round cell sarcomas, the main candidate would be desmoplastic small round cell tumor, although this neoplasm is distinguishable from EWS/PNET in most cases on the basis of immunohistochemical differences. Nonetheless, as exemplified by the undifferentiated round cell sarcoma category in this study, some cases do not fit neatly into a single diagnostic category. Since FISH fusion, FISH break apart, and RT-PCR are all useful and complementary techniques, more than one technique may be needed to clarify the most diagnostically challenging cases. 


\section{References}

1 Toskos M. Peripheral primitive neuroectodermal tumors. Diagnosis, classification, and prognosis. Perspect Pediatr Pathol 1992;16:27-98.

2 Parham D, Rolosoin G, Feely M, et al. Primary malignant neuroepithelial tumors of the kidney. Am J Sur Pathol 2001;25:133-146.

3 Aurias A, Rimbaut C, Buffe C, et al. Chromosomal translocations in Ewing's sarcoma. $\mathrm{N}$ Engl J Med 1983;309:496-497.

4 Turc-Carel C, Philip I, Berge MP, et al. Chromosomal translocations in Ewing's sarcoma. $\mathrm{N}$ Engl J Med 1983;309:497-498.

5 Whang-Peng J, Triche TJ, Knutsen T, et al. Chromosome translocation in peripheral neuroepithelioma. $\mathrm{N}$ Engl J Med 1984;311:584-585.

6 Turc-Carel C, Aurias A, Mugneret F, et al. Chromosomes in Ewing's sarcoma. I. An evaluation of 85 cases and remarkable consistency of $\mathrm{t}(11 ; 22) \mathrm{q} 24 ; \mathrm{q} 12)$. Cancer Genet Cytogenet 1988;32:229-238.

7 Aurias A, Rimbaut C, Buffe C, et al. Translocation involving chromosome 22 in Ewing's sarcoma. A cytogenetic study of four fresh tumors. Cancer Genet Cytogenet 1984;12:21-25.

8 Whang-Peng J, Triche TJ, Knutsen T, et al. Cytogenetic characterization of selected small round cell tumors of childhood. Cancer Genet Cytogenet 1986;21:185-208.

9 Shing DC, McMullan DJ, Roberts P, et al. FUS/ERG gene fusions in Ewing's tumors. Cancer Res 2003;63: 4568-4576.

10 Delattre O, Zucman J, Plougastel B, et al. Gene fusion with an ETS DNA-binding domain caused by chromosome translocation in human tumours. Nature 1992; 359:162-165.

11 Delattre O, Zucman J, Melot T, et al. The Ewing family of tumors: a subgroup of small round cell tumors defined by specific chimeric transcripts. N Engl J Med 1994;331:294-299.

12 Downing JR, Head DR, Parham DM, et al. Detection of the $\mathrm{t}(11 ; 22)(\mathrm{q} 24-\mathrm{q} 12)$ translocation of Ewing's sarcoma and peripheral neuroectodermal tumor by reverse transcription polymerase chain reaction. Am J Pathol 1993;143:1294-1300.

13 Desmaze C, Zucman J, Delattre O, et al. Unicolor and bicolor in situ hybridization in the diagnosis of peripheral neuroepithelioma and related tumors. Genes Chrom Cancer 1992;5:30-34.

14 Taylor C, Patel C, Jones T, et al. Diagnosis of Ewing's sarcoma and peripheral neuroectodermal tumour based on the detection of $t(11 ; 22)$ using fluorescence in situ hybridization. Br J Cancer 1993;67:128-133.

15 McManus AP, Gusterson BA, Pinkerton CR, et al. Diagnosis of Ewing's sarcoma and related tumours by detection of chromosome 22q12 translocations using fluorescence in situ hybridizations on tumour touch imprints. J Pathol 1995;176:137-142.

16 Desmaze C, Zucman J, Delattre O, et al. Interphase molecular cytogenetics of Ewing's sarcoma and peripheral neuroepithelioma $\mathrm{t}(11 ; 22)$ with flanking and overlapping cosmid probes. Cancer Genet Cytogenet 1994;74:13-18.

17 Desmaze C, Aurias A. In situ hybridization of fluorescent probes on chromosomes, nuclei or stretched DNA: applications in physical mapping and characterization of genomic rearrangements. Cell Mol Biol. 1995;141:925-931.
18 Qian X, Jin L, Shearer BM, et al. Molecular diagnosis of Ewing's sarcoma/primitive neuroectodermal tumor in formalin-fixed paraffin-embedded tissues by RT-PCR and fluorescence in situ hybridization. Diagn Mol Pathol 2005;14:23-28.

19 Perry A, Aldape KD, George DH, et al. Small cell astrocytoma: an aggressive variant that is clinicopathologically and genetically distinct from anaplastic oligodendroglioma. Cancer 2004;101:2318-2326.

20 Kumar S, Pack S, Kumar D, et al. Detection of EWSFLI-1 fusion in Ewing's sarcoma/peripheral primitive neuroectodermal tumor by fluorescence in situ hybridization using formalin-fixed paraffin-embedded tissue. Hum Pathol 1999;30:324-330.

21 Hill DA, O’Sullivan MJ, Zhu X, et al. Practical application of molecular genetic testing as an aid to the surgical pathologic diagnosis of sarcomas. A prospective study. Am J Surg Pathol 2002;26: 965-977.

22 O’Sullivan MJ, Perlman EJ, Furman J, et al. Visceral primitive peripheral neuroectodermal tumors: a clinicopathologic and molecular study. Hum Pathol 2001;32:1109-1115.

23 Teitell MA, Thompson AD, Sorensen PHB, et al. EWS/ ETS fusion genes induce epithelial and neuroectodermal differentiation in NIH 3T3 fibroblasts. Lab Invest 1999;79:1535-1543.

24 Barnoud R, Sabourin JC, Pasquier D, et al. Immunohistochemical expression of WT1 by desmoplastic small round cell tumor: a comparative study with other small round cell tumors. Am J Surg Pathol 2000;24:830-836.

25 Devoe K, Weidner N. Immunohistochemistry of small round-cell tumors. Sem Diagn Pathol 2000;17: 216-224.

26 Folpe AL, Hill CE, Parham DM, et al. Immunohistochemical detection of FLI-1 protein expression: a study of 132 round cell tumors with emphasis on CD99positive mimics of Ewing sarcoma/primitive neuroectodermal tumor. Am J Surg Pathol 2000;24:1657-1662.

27 Llombart-Bosch A, Navarro S. Immunohistochemical detection of EWS and FLI-1 proteins in Ewing sarcoma and primitive neuroectodermal tumors: comparative analysis with CD99 (MIC-2) expression. Appl Immunohistochem Mol Morphol 2001;9:255-260.

28 Cessna $\mathrm{MH}$, Zhou H, Perkins SL, et al. Are myogenin and MyoD1 expression specific for rhabdomyosarcoma? A study of 150 cases, with emphasis on spindle cell mimics. Am J Surg Pathol 2001;25:1150-1157.

29 Carpentieri DF, Nichols K, Chou PM, et al. The expression of WT1 in the differentiation of rhabdomyosarcoma from other pediatric small round blue cell tumors. Mod Pathol 2002;15:1080-1086.

30 Zhang PJ, Goldblum JR, Pawel BR, et al. Immunophenotype of desmoplastic small round cell tumors as detected in cases with EWS-WT1 gene fusion product. Mod Pathol 2003;16:229-235.

31 Rossi S, Orvieto A, Furlanetto A, et al. Utility of the immunohistochemical detection of FLI-1 expression in round cell and vascular neoplasm using a monoclonal antibody. Mod Pathol 2004;17:547-552.

32 Lae ME, Roche PC, Jin L, et al. Desmoplastic small round cell tumor. A clinicopathologic, immunohistochemical, and molecular study of 32 tumors. Am J Surg Pathol 2002;26:823-835.

33 Zucman J, Delattre O, Desmaze C, et al. EWS and ATF-1 gene fusion induced by $\mathrm{t}(12 ; 22)$ translocation in 
malignant melanoma of soft parts. Nat Genet 1993;4: 341-345.

34 Clark J, Benjamin H, Gill S, et al. Fusion of the EWS gene to CHN, a member of the steroid/thyroid receptor gene superfamily, in a human myxoid chondrosarcoma. Oncogene 1996;12:229-235.

35 Zucman J, Melot T, Desmaze C, et al. Combinatorial generation of variable fusion proteins in the Ewing family of tumours. EMBO J 1993;12:4481-4487.
36 Zoubek A, Dockhorn-Dworniczak B, Delattre O, et al. Does expression of different EWS chimeric transcripts define clinically distinct risk groups of Ewing tumor patients? J Clin Oncol 1996;14: 1245-1251.

37 de Alava E, Kawai A, Healey JH, et al. EWS-FLI1 fusion transcript structure is an independent determinant of prognosis in Ewing's sarcoma. J Clin Oncol 1988;16:1248-1255. 